\title{
Tumor Size and Lobular Histology Predict for Higher Pathologic Nodal Stage Regardless of Clinical Presentation of Nodal Disease in Patients With Breast Cancer
}

\section{Linda Ye}

University of California Los Angeles

Dennis Rünger

University of california los angeles

Stephanie A. Angarita

Kaiser Permanente Medical Center Joseph Hadaya

University of California Los Angeles

Jennifer L. Baker

University of California Los Angeles

Minna K. Lee

Memorial Sloan Kettering Cancer Center

Carlie K. Thompson

university of california los angeles

\section{Deanna J. Attai}

university of california los angeles

Maggie DiNome ( $\square$ maggie.dinome@duke.edu )

Duke-NUS: Duke-NUS Medical School https://orcid.org/0000-0002-1926-292X

\section{Research Article}

Keywords: nodal stage, palpable adenopathy, clinically node positive, lobular histology, breast cancer

Posted Date: November 9th, 2021

DOI: https://doi.org/10.21203/rs.3.rs-1032846/v1

License: (c) (i) This work is licensed under a Creative Commons Attribution 4.0 International License. Read Full License 


\section{Abstract}

Purpose: Omission of axillary lymph node dissection (ALND) is considered for patients with sentinel lymph node-positive (SLN+) breast cancer, but ALND remains the standard of care for clinically nodepositive $(\mathrm{cN}+)$ patients treated with surgery first. Here, we evaluate differences in patient and tumor characteristics and pathologic nodal stage in patients with positive lymph nodes who underwent ALND.

Methods: Retrospective chart review from 2010-2019 identified three groups of patients who underwent ALND for positive nodes: SLN+ (positive node identified at SLN biopsy), cNUS (abnormal preoperative US and biopsy), and cNpalp (palpable adenopathy). Patients who received neoadjuvant chemotherapy or presented with axillary recurrence were excluded.

Results: Of 218 patients, 107 were SLN+, 43 were cNUS, and 68 were cNpalp. SLN+ patients compared with cNpalp were more likely to be younger (56 vs $64, p<.01)$, pre-menopausal (39\%vs $15 \%, p<.01)$, and white $(62 \% \mathrm{vs} 37 \%, p<.01)$ with more tumors that were progesterone receptor-positive $(6 \% \mathrm{vs} 21 \%, p=.02)$, low grade $(35 \% \mathrm{vs} 6 \%, p<.01)$ and without lymphovascular invasion $(11 \% \mathrm{vs} 27 \%, p=.02)$. SLN+ patients had more pN1 disease than cNUS and cNpalp $(67.3 \%$ vs $39.5 \%$ vs $42.6 \%, p<.01)$. Greater tumor size and lobular histology were significantly associated with higher nodal stage in univariable regression analysis of SLN+ patients as well as a pooled analysis of the three clinical groups.

Conclusion: Patient and tumor characteristics differ on either end of the nodal spectrum, with cNpalp patients having higher risk features than SLN+ patients. These higher risk features have historically resulted in ALND for patients with clinically positive nodes. However, only tumor size and histology are associated with higher pathologic nodal stage.

\section{Introduction}

Axillary lymph node dissection (ALND) was once considered the standard of care for management of the axilla in patients with breast cancer for prevention of metastatic spread. Now, axillary surgery is understood to be more of a staging procedure than for treatment and cure. In fact, both the ACOSOG Z0011 ${ }^{1}$ and AMAROS ${ }^{2}$ trials demonstrated that disease left undissected in the axilla in up to $27 \%$ and $33 \%$ of patients, respectively, did not worsen rates of locoregional recurrence or survival compared to patients who underwent completion ALND. Based on these data, omission of ALND is accepted for patients with clinically negative axillae who are found to have 1-2 positive nodes at sentinel lymph node biopsy (SLNB) who undergo breast conservation therapy. The NCCN also recently updated their guidelines (version 8.2021) to allow patients with image-detected metastases to undergo SLNB for surgical staging. However, ALND remains the standard of care for patients with clinically palpable adenopathy who proceed directly to surgery and are not downstaged with neoadjuvant chemotherapy (NAC).

Studies comparing patients with sentinel node-positive (SLN+) disease to those with lymph node metastases diagnosed by preoperative ultrasound and biopsy (cNUS) suggest that cNUS patients have 
higher risk features and greater pathologic nodal burden ${ }^{3-6}$. Few studies have evaluated patients with palpable adenopathy (cNpalp), but the assumption exists that increasing clinical nodal burden reflects higher risk features and greater pathologic nodal disease. In this present study, we compare preoperative patient and tumor characteristics of patients with breast cancer who underwent ALND for positive nodal disease (SLN+, cNUS, cNpalp) and assess for variables associated with higher pathologic nodal stage among these different patient groups.

\section{Methods}

This study was approved by the Institutional Review Board of the University of California, Los Angeles.

\section{Study Design}

This is a retrospective study of patients who underwent ipsilateral ALND for breast cancer between 20102019 at a single institution. Consecutive patients were identified from chart review based on Current Procedural Terminology (CPT) codes and International Classification of Diseases, Ninth and Tenth Revision (ICD 9/10) diagnosis codes. Patients were excluded if they were treated with NAC, presented with stage 4 disease, had matted nodes on exam, or underwent ALND for axillary recurrence or contralateral axillary disease.

Patients were categorized into three clinical groups: clinically negative axilla but positive sentinel lymph nodes identified on SLNB (SLN+), non-palpable but positive lymph nodes on preoperative ultrasound and biopsy (cNUS), and palpable lymphadenopathy on preoperative exam (cNpalp). We considered cNUS and cNpalp patients to have clinically node-positive $(\mathrm{cN}+)$ disease. Ipsilateral SLNB was performed in patients with invasive breast cancer and clinically negative axillae who underwent lumpectomy or mastectomy. Positive sentinel lymph node status was defined as micro- or macro-metastatic disease in at least one node on final surgical pathology.

Patient demographic and tumor characteristics, type of breast and axillary surgery, and adjuvant therapies received were collected from medical records. The presence of clinically positive nodes was assessed from documentation of preoperative clinical exam, imaging, and pathology results. Clinical tumor size was defined by imaging or exam before surgery based on standard American Joint Commission on Cancer (AJCC) staging criteria. To provide predictive guidance of nodal status prior to surgery, tumor histology, nuclear grade, prognostic markers [estrogen receptor (ER), progesterone receptor (PR), human epidermal growth factor receptor 2/neu (HER2neu) status], Ki67 levels, and presence of lymphovascular invasion (LVI) were captured from the preoperative core needle biopsy results of the primary breast cancer.

The total number of positive lymph nodes was determined from the final surgical pathology specimen. Lymph node burden was defined by pathologic nodal stage as pN1 (1-3 positive nodes), pN2 (4-9 positive nodes), or pN3 ( $\geq 10$ positive nodes). 


\section{Statistical Analysis}

Descriptive statistics on patient, tumor, and nodal characteristics were compiled by clinical group or nodal stage and assessed using analysis of variance for continuous variables and Fisher's exact test for categorical variables. Post hoc pairwise comparisons were performed using Tukey's test or Fisher's exact test with Benjamini-Hochberg correction for multiple testing. The R software environment version 4.0.2 was used for all statistical analyses. Two-sided $p<.05$ was considered significant.

\section{Results}

\section{Patient and Tumor Characteristics by Nodal Group}

Table 1 describes the preoperative demographic and tumor characteristics of all patients who underwent ALND compared across the three clinical groups. Of 218 patients total, $107(49.1 \%)$ were SLN+, 43 $(19.7 \%)$ were cNUS, and the remaining 68 (31.2\%) were cNpalp. The overall mean age was 59.3 years, with most patients being female $(99.1 \%)$ and post-menopausal $(70.1 \%)$, with tumors predominantly ERpositive (94.1\%) and of ductal histology (72.9\%). 
Table 1

Patient Demographic and Tumor Characteristics Stratified by Clinical Nodal Group

\begin{tabular}{|c|c|c|c|c|c|}
\hline \multirow[t]{3}{*}{ Characteristic } & \multirow{3}{*}{$\begin{array}{l}\text { Overalla }^{a} \\
(n=218)\end{array}$} & \multicolumn{4}{|c|}{ Clinical nodal group } \\
\hline & & SLN+ & cNUS & cNpalp & $\mathrm{p}$ value \\
\hline & & $(n=107)$ & $(n=43)$ & $(n=68)$ & \\
\hline Age $(y r s)$, mean $\pm S D$ & $59.3 \pm 12.4$ & $56.9 \pm 12.3$ & $58.3 \pm 10.5$ & $63.7 \pm 12.7$ & $<.01$ \\
\hline Sex at birth & & & & & 1 \\
\hline Male & $2(0.9)$ & $1(0.9)$ & $0(0.0)$ & $1(1.5)$ & \\
\hline Female & $215(99.1)$ & $105(99.1)$ & $43(100.0)$ & $67(98.5)$ & \\
\hline Race/ethnicity & & & & & $<.01$ \\
\hline White & $108(49.8)$ & $66(62.3)$ & $17(39.5)$ & $25(36.8)$ & \\
\hline Hispanic & $18(8.3)$ & $7(6.6)$ & $0(0.0)$ & $11(16.2)$ & \\
\hline Black & $8(3.7)$ & $2(1.9)$ & $3(7.0)$ & $3(4.4)$ & \\
\hline Asian & $25(11.5)$ & $11(10.4)$ & $7(16.3)$ & $7(10.3)$ & \\
\hline Other & $58(26.7)$ & $20(18.9)$ & $16(37.2)$ & $22(32.4)$ & \\
\hline Menopausal status & & & & & $<.01$ \\
\hline Pre & $64(29.9)$ & $41(39.0)$ & $13(30.2)$ & $10(15.2)$ & \\
\hline Post & $150(70.1)$ & $64(61.0)$ & $30(69.8)$ & $56(84.8)$ & \\
\hline Positive nodes & & & & & $<.01$ \\
\hline $1-3$ (pN1) & $118(54.1)$ & $72(67.3)$ & $17(39.5)$ & $29(42.6)$ & \\
\hline $4+(p N 2+)$ & $100(45.9)$ & $35(32.7)$ & $26(60.5)$ & $39(57.4)$ & \\
\hline Histology & & & & & .72 \\
\hline Ductal & $153(72.9)$ & $76(73.1)$ & $28(68.3)$ & $49(75.4)$ & \\
\hline Lobular & $48(22.9)$ & $25(24.0)$ & $10(24.4)$ & $13(20.0)$ & \\
\hline Other & $9(4.3)$ & $3(2.9)$ & $3(7.3)$ & $3(4.6)$ & \\
\hline
\end{tabular}

Bold values indicate $p<.05$

$S L N+$ clinically negative, sentinel lymph node-positive disease; $c N U S$ clinically positive, non-palpable, US+ nodal disease; $c N p a l p$ clinically positive, palpable nodal disease; $E R$ estrogen receptor; $P R$ progesterone receptor; $L V I$ lymphovascular invasion.

aUnless otherwise indicated, data are expressed as number (percentage) of patients. Percentages have been rounded and may not total 100 . 


\begin{tabular}{|c|c|c|c|c|c|}
\hline \multirow[t]{3}{*}{ Characteristic } & \multirow{3}{*}{$\begin{array}{l}\text { Overalla }^{a} \\
(n=218)\end{array}$} & \multicolumn{4}{|c|}{ Clinical nodal group ${ }^{a}$} \\
\hline & & SLN+ & cNUS & cNpalp & $p$ value \\
\hline & & $(n=107)$ & $(n=43)$ & $(n=68)$ & \\
\hline \multicolumn{5}{|l|}{ ER stain } & .12 \\
\hline 0 & $12(5.9)$ & $3(3.1)$ & $2(4.8)$ & 7 (10.9) & \\
\hline $1+$ & $191(94.1)$ & $94(96.9)$ & $40(95.2)$ & 57 (89.1) & \\
\hline \multicolumn{5}{|l|}{ PR stain } & .02 \\
\hline 0 & $24(12.0)$ & $6(6.2)$ & $5(11.9)$ & $13(21.0)$ & \\
\hline $1+$ & $176(88.0)$ & $90(93.8)$ & $37(88.1)$ & $49(79.0)$ & \\
\hline \multicolumn{5}{|l|}{ Tumor grade } & $<.01$ \\
\hline 1 & $46(23.4)$ & $34(35.1)$ & $8(21.1)$ & $4(6.5)$ & \\
\hline 2 & $104(52.8)$ & $47(48.5)$ & $22(57.9)$ & 35 (56.5) & \\
\hline 3 & 47 (23.9) & $16(16.5)$ & $8(21.1)$ & $23(37.1)$ & \\
\hline \multicolumn{5}{|l|}{ Ki67 } & .02 \\
\hline$<15 \%$ & $70(40.5)$ & 41 (51.9) & $16(42.1)$ & $13(23.2)$ & \\
\hline $15-35 \%$ & $63(36.4)$ & $24(30.4)$ & $13(34.2)$ & $26(46.4)$ & \\
\hline$>35 \%$ & $40(23.1)$ & $14(17.7)$ & $9(23.7)$ & $17(30.4)$ & \\
\hline \multicolumn{5}{|l|}{ Tumor size } & .33 \\
\hline $\mathrm{T} 1(\leq 20 \mathrm{~mm})$ & 70 (33.2) & $41(39.0)$ & $12(28.6)$ & $17(26.6)$ & \\
\hline T2 (21-50 mm) & 101 (47.9) & $44(41.9)$ & $24(57.1)$ & $33(51.6)$ & \\
\hline T3 (> $50 \mathrm{~mm})$ & $40(19.0)$ & $20(19.0)$ & $6(14.3)$ & $14(21.9)$ & \\
\hline \multicolumn{5}{|l|}{ LVI } & .02 \\
\hline Present & 35 (18.3) & $10(10.6)$ & $9(23.7)$ & $16(27.1)$ & \\
\hline Absent & 156 (81.7) & $84(89.4)$ & $29(76.3)$ & 43 (72.9) & \\
\hline \multicolumn{6}{|c|}{ Bold values indicate $p<.05$. } \\
\hline \multicolumn{6}{|c|}{$\begin{array}{l}S L N+\text { clinically negative, sentinel lymph node-positive disease; } c N U S \text { clinically positive, non-palpable, } \\
\text { US+ nodal disease; } c N p \text { alp clinically positive, palpable nodal disease; } E R \text { estrogen receptor; } P R \\
\text { progesterone receptor; } L V I \text { lymphovascular invasion. }\end{array}$} \\
\hline
\end{tabular}


The three patient groups showed significant differences on all demographic and tumor characteristics except for histology, ER-positivity, and tumor size. These differences were investigated further with multiple pairwise group comparisons (Figure 1). For race/ethnicity, more patients identified as White in the SLN+ group compared with the cNUS $(62.3 \%$ vs. $39.5 \%, p=.03)$ and cNpalp $(62.3 \%$ vs. $36.8 \%, p<.01)$ groups, with no difference noted between cNUS versus cNpalp $(p=.84)$ patients. Similarly, rates of pN1 disease were significantly different between SLN+ versus cNUS $(67.3 \%$ vs. $39.5 \%, p<.01)$ and SLN+ versus cNpalp $(67.3 \%$ vs. $42.6 \%, p<.01)$ but not between cNUS versus cNpalp $(p=.84)$ patients. SLN+ patients were younger $(-6.79$ years, [95\% $\mathrm{Cl}-11.21--2.36], p<.01)$ and more often pre-menopausal $(39.0 \%$ vs. $15.2 \%, p<.01)$ compared to cNpalp but not cNUS patients.

Regarding tumor characteristics, a progression from lower risk features in the SLN+ group to higher risk features in the cNpalp group was noted. Significant differences were obtained when comparing SLN+ patients and cNpalp patients on PR+ $(93.8 \%$ vs. $79.0 \%, p=.03)$, low tumor grade $(35.1 \%$ vs. $6.5 \%, p<.01)$, low Ki67 (51.9\% vs. $23.2 \%, p=.01)$, and presence of LVI $(10.6 \%$ vs. $27.1 \%, p=.02)$. The intermediate cNUS group did not differ significantly from the SLN+ or the cNpalp group.

When we restricted the study sample to ER+ patients only, the sample size was reduced to 191, and the effects for PR, Ki67, and LVI were no longer significant (with p values slightly above .05). Excluding ER(-) patients rendered the nodal groups more statistically similar due to the homogenization and/or reduction in sample size.

\section{Predictors of Higher Pathologic Nodal Stage in SLN+ Patients}

Preoperative demographic and tumor characteristics of patients with SLN+ disease who underwent ALND were stratified by nodal stage in Table 2. Of the 107 patients with SLN+ disease, 72 (67.3\%) were found to have pN1 disease on final pathology, while 25 (23.4\%) had pN2 disease, and 10 (9.3\%) had pN3 disease. The mean age was 56.9 years, with most patients being female $(99.1 \%)$ and post-menopausal $(61.0 \%)$ with ER-positive disease (96.9\%). The predominant histological subtype was ductal (73.1\%), with most patients presenting with either T1 $(35.1 \%)$ or T2 $(48.5 \%)$ disease. About $2 / 3$ of patients $(67.3 \%)$ had $\leq 3$ positive nodes on final pathology. On univariable analysis, larger tumor size $(p=.02)$ and lobular histology $(p=.01)$ were found to be significantly associated with greater nodal burden. Post hoc comparisons showed that patients with pN3 disease differed significantly from patients with pN1 disease on tumor size $(p=.01)$. For histology, there was a significant difference between pN3 and pN1 patients ( $p$ $=.01)$, with the difference between $\mathrm{pN} 3$ and $\mathrm{pN} 2$ falling short of significance $(p=.06)$. Age, sex, race/ethnicity, menopausal status, tumor grade, Ki67 level, and presence of LVI did not differ with nodal stage. 
Table 2

\begin{tabular}{|c|c|c|c|c|c|}
\hline \multirow[t]{3}{*}{ Characteristic } & \multirow{3}{*}{$\begin{array}{l}\text { Overalla }^{a} \\
(n=107)\end{array}$} & \multicolumn{3}{|c|}{ Number of positive nodes ${ }^{a}$} & \multirow[t]{3}{*}{$p$ value } \\
\hline & & $1-3(\mathrm{pN} 1)$ & 4-9 (pN2) & $10+(\mathrm{pN} 3)$ & \\
\hline & & $(n=72)$ & $(n=25)$ & $(n=10)$ & \\
\hline Age (yrs), mean \pm SD & $56.9 \pm 12.3$ & $56.4 \pm 11.6$ & $56.2 \pm 14.0$ & $63.0 \pm 12.9$ & .26 \\
\hline Sex at birth & & & & & 1 \\
\hline Male & $1(0.9)$ & $1(1.4)$ & $0(0.0)$ & $0(0.0)$ & \\
\hline Female & 105 (99.1) & 71 (98.6) & $24(100.0)$ & $10(100.0)$ & \\
\hline Race/ethnicity & & & & & .54 \\
\hline White & $66(62.3)$ & $41(57.7)$ & $17(68.0)$ & $8(80.0)$ & \\
\hline Hispanic & $7(6.6)$ & $3(4.2)$ & $3(12.0)$ & $1(10.0)$ & \\
\hline Black & $2(1.9)$ & $2(2.8)$ & $0(0.0)$ & $0(0.0)$ & \\
\hline Asian & $11(10.4)$ & $10(14.1)$ & $1(4.0)$ & $0(0.0)$ & \\
\hline Other & $20(18.9)$ & $15(21.1)$ & $4(16.0)$ & $1(10.0)$ & \\
\hline Menopausal status & & & & & .47 \\
\hline Pre & $41(39.0)$ & $28(40.0)$ & $11(44.0)$ & $2(20.0)$ & \\
\hline Post & $64(61.0)$ & $42(60.0)$ & $14(56.0)$ & $8(80.0)$ & \\
\hline Histology & & & & & .01 \\
\hline Ductal & $76(73.1)$ & $55(79.7)$ & $18(72.0)$ & $3(30.0)$ & \\
\hline Lobular & $25(24.0)$ & $13(18.8)$ & $6(24.0)$ & $6(60.0)$ & \\
\hline Other & $3(2.9)$ & $1(1.4)$ & $1(4.0)$ & $1(10.0)$ & \\
\hline Tumor grade & & & & & .66 \\
\hline 1 & $34(35.1)$ & $20(31.7)$ & $11(45.8)$ & $3(30.0)$ & \\
\hline 2 & $47(48.5)$ & $31(49.2)$ & $11(45.8)$ & $5(50.0)$ & \\
\hline 3 & $16(16.5)$ & $12(19.0)$ & $2(8.3)$ & $2(20.0)$ & \\
\hline
\end{tabular}

Bold values indicate $p<.05$.

LVIlymphovascular invasion.

aUnless otherwise indicated, data are expressed as number (percentage) of patients. Percentages have been rounded and may not total 100 . 


\begin{tabular}{|c|c|c|c|c|c|}
\hline \multirow[t]{3}{*}{ Characteristic } & \multirow{3}{*}{$\begin{array}{l}\text { Overalla }^{a} \\
(n=107)\end{array}$} & \multicolumn{3}{|c|}{ Number of positive nodes ${ }^{a}$} & \multirow[t]{3}{*}{$p$ value } \\
\hline & & $1-3(\mathrm{pN} 1)$ & 4-9 (pN2) & $10+(p N 3)$ & \\
\hline & & $(n=72)$ & $(n=25)$ & $(n=10)$ & \\
\hline Ki67 & & & & & .44 \\
\hline$<15 \%$ & $41(51.9)$ & $26(49.1)$ & $10(55.6)$ & $5(62.5)$ & \\
\hline $15-35 \%$ & $24(30.4)$ & $16(30.2)$ & $7(38.9)$ & $1(12.5)$ & \\
\hline$>35 \%$ & $14(17.7)$ & $11(20.8)$ & $1(5.6)$ & $2(25.0)$ & \\
\hline Tumor size & & & & & .02 \\
\hline $\mathrm{T} 1(0-20 \mathrm{~mm})$ & $41(39.0)$ & $27(38.6)$ & $11(44.0)$ & $3(30.0)$ & \\
\hline T2 (21-50 mm) & $44(41.9)$ & $34(48.6)$ & $9(36.0)$ & $1(10.0)$ & \\
\hline $\mathrm{T} 3(>50 \mathrm{~mm})$ & $20(19.0)$ & 9 (12.9) & $5(20.0)$ & $6(60.0)$ & \\
\hline LVI & & & & & .2 \\
\hline Present & $10(10.6)$ & $4(6.6)$ & $4(17.4)$ & $2(20.0)$ & \\
\hline Absent & $84(89.4)$ & $57(93.4)$ & $19(82.6)$ & $8(80.0)$ & \\
\hline \multicolumn{6}{|c|}{ Bold values indicate $p<.05$. } \\
\hline \multicolumn{6}{|c|}{ LVIlymphovascular invasion. } \\
\hline
\end{tabular}

\section{Predictors of Higher Pathologic Nodal Stage in the Pooled Sample of SLN+ and $\mathrm{cN}+$ Patients}

Tumor size and lobular histology were also previously found to be significant predictors of higher pathologic nodal stage in the cohort of patients with clinically positive nodes ${ }^{7}$. Given the similar pattern of associations among SLN+ and cN+ patients, we combined the nodal groups to examine these associations in a larger sample $(n=218)$. Univariable analysis of the pooled cohort confirmed that larger tumor size and lobular histology were predictive of higher nodal burden (both $p s<.01$ ). In post hoc pairwise comparisons, pN3 patients differed in tumor size from pN1 patients $(p<.01)$ as well as pN2 patients $(p<.01)$. For histology, there was a significant difference between pN3 and pN1 patients $(p<$ .01). By contrast, age, sex, race/ethnicity, menopausal status, tumor grade, Ki67 level, and presence of LVI were again unrelated to pathologic nodal stage despite the increase in statistical power (all $p s>.13$ ). Subgroup analysis of the $E R+$ patient cohort $(n=191)$ similarly demonstrated that only histology $(p<.01)$ and tumor size $(p=.02)$ were significantly associated with nodal stage (all other $p s>.11)$. 
A multivariable logistic regression of pN1 disease in the pooled sample with histology and tumor size as predictors found that patients with $\mathrm{T} 3$ tumors had 70\% lower odds of having pN1 disease than patients with $\mathrm{T} 1$ tumors $(\mathrm{OR}=0.30,95 \% \mathrm{Cl}[0.12,0.72], \mathrm{p}=.01)$. The effect of histology was not significant $(\mathrm{p}=$ .47).

\section{Surgical Treatment, Adjuvant Therapies, and Outcomes}

Overall, 73.4\% (160/218) of patients underwent mastectomy in this study cohort of node positive disease with no difference in numbers of mastectomies performed between the $\mathrm{SLN}+(79 / 160)$ and $\mathrm{cN}+(81 / 160)$ groups $(p=1)$. Receipt of adjuvant radiation therapy $(p=0.48)$ or systemic chemotherapy $(p=0.64)$ did not differ between patients who presented with $\mathrm{SLN}+$ or $\mathrm{CN}+$ disease. However, significantly more patients with SLN+ disease (94.2\%) received endocrine therapy compared to patients with $\mathrm{cN}+$ disease $(75.7 \%)(p<.01)$.

Of patients with pN1 disease (118/218), 70.3\% (83/118) were treated with mastectomy. Of those, $69.6 \%$ received post-mastectomy and regional nodal irradiation. Over half of patients with pN1 disease (56.2\%) received both chemotherapy and endocrine therapy, which did not differ based on nodal presentation $(p=$ $0.25)$.

\section{Discussion}

Historic randomized study data have failed to demonstrate a survival benefit for axillary lymph node dissection for patients with clinically positive nodes ${ }^{8-10}$, yet ALND has remained the standard of care for these patients. Recent practice-changing clinical trials assessing de-escalation of axillary surgery have excluded patients with clinically positive nodes ${ }^{1,2}$, resulting in a significant dearth of contemporary data to guide surgical management of the axilla in this patient cohort. More recently, nodal downstaging with NAC has become an acceptable strategy to avoid ALND for patients with clinically positive nodes who respond clinically to treatment ${ }^{11-13}$, but nodal response rates vary considerably by tumor subtype with the highest rates seen in patients with Her2-overexpressing (Her2+) or triple negative breast cancer $(T N B C)^{14-16}$. For patients with ER-positive disease, nodal downstaging rates rarely exceed $20 \%$, and since residual disease is not prognostic of outcome for this cancer subtype ${ }^{15,17,18}$, patients are typically recommended for surgery first, and often ALND.

The concern for de-escalation of axillary surgery for patients with clinically positive nodes appears to stem from data that demonstrate that these patients have less favorable tumor characteristics than patients with $\mathrm{SLN}+$ disease $^{3-6}$. Our data of primarily ER-positive patients support these findings and demonstrate that progression of tumor characteristics from lower to higher risk mirrors the progression of clinical nodal disease from SLN+ to cNUS to cNpalp, with statistically significant differences existing at both ends of the nodal spectrum. Patients with clinically palpable nodes have lower PR-positivity, higher tumor grade, higher Ki67 levels, and higher rates of LVI than patients with SLN+ disease, but these higher risk features do not predict for higher nodal stage. When we limit our study group to ER+ patients only, we 
note fewer clinicopathologic group differences, which may reflect that the differences in risk features are driven by the ER- breast cancers and that there is more homogeneity among ER+ breast cancers despite nodal presentation. However, we are cautious to interpret this lack of significant difference as evidence for the absence of a difference, especially when the effect fell out of significance after a reduction in sample size. Regardless, in both the overall cohort and the ER+ subgroup, only tumor size and lobular histology appear associated with higher pathologic nodal burden among patients with node positive disease.

Previous studies support our findings that size of the primary tumor and non-ductal histology are the pertinent factors associated with higher pathologic nodal stage in patients with both SLN+ and cNUS breast cancer ${ }^{5,19-22}$. Caudle et al ${ }^{5}$ compared over 700 patients who had either SLN+ or cNUS-detected disease but excluded patients with palpable adenopathy, and similarly identified that age, grade, and PRpositivity differed between the two groups, but these higher risk features did not predict for higher nodal stage. Only tumor size and lobular histology were related to extent of nodal disease, and this did not differ between SLN+ or cNUS patients. Unlike the present study, the authors demonstrated that the method of nodal presentation, i.e. having metastases identified by US (odds ratio [OR] 3.80, [95\% $\mathrm{Cl} 2.65$ - 5.43]; $p$.0001) was correlative of $\geq 3$ positive nodes. However, it is unclear whether this variable would be predictive of pathologic nodal upstaging (> pN1 disease). In addition, only $77.9 \%$ of patients in the cNUS group were ER-positive, which was statistically different than the SLN+ group, and $14 \%$ were Her2+. In a study by Mamtani et $\mathrm{al}^{20}$, in which patients with SLN+ disease were evaluated, only tumor size and lobular histology were independently associated with additional positive nodes on ALND when adjusting for ER and Her2 status. Grade and presence of LVI did not appear to influence nodal positivity.

Other studies have demonstrated that certain high-risk features do predict for higher nodal stage, which led authors to conclude that patients with clinically positive nodes should not be considered for omission of $\mathrm{ALND}^{3-6}$. However, a disproportionately high number of patients in these studies had ER-negative (23.9\%-29.7\%) or Her2-positive (18.7\%-31.2\%) disease. Modern day treatment paradigms would likely include neoadjuvant chemotherapy for this patient cohort with node-positive, ER-negative or Her2-positive disease, which make these datasets less reliable for guiding surgical management recommendations for the primarily ER-positive breast cancer patients who proceed straight to surgery.

Few studies have evaluated nodal burden in patients with palpable adenopathy and ER-positive, Her2negative breast cancer, in whom downstaging with NAC is less effective and alternatives to ALND are needed. Angarita et al. ${ }^{7}$ performed a subgroup analysis of this specific patient population and demonstrated that $44.6 \%$ of patients had pN1 disease, and tumor size and lobular histology were significant predictors of $>\mathrm{pN} 1$ disease. Crown et al ${ }^{22}$ similarly demonstrated that $57 \%$ of patients with palpable adenopathy had pathologically low nodal burden (pN1). More than $97 \%$ of patients in that study had ER-positive disease, and only tumor size and non-ductal histology were again associated with higher nodal stage. Higher risk features such as palpable adenopathy, LVI and high tumor grade did not predict for higher nodal stage. 
Certainly, it is understood that higher risk features carry worsened prognoses, but this finding should not obligate patients to extensive lymphatic axillary surgery, particularly since randomized trial data have not demonstrated a survival benefit to $A L N D^{8-10}$. With the risk of disabling lymphedema and functional morbidity accompanying this surgical procedure, the oncologic benefit to ALND needs to be continually assessed for all patients. Verheuvel et al. ${ }^{4}$ demonstrated a worsened overall (hazard ratio [HR] 2.67, [95\% $\mathrm{Cl} 1.48$ - 4.84]) and disease-free survival (HR 2.71, [95\% Cl 1.49 - 4.92]) in patients with cNUS positive disease compared to SLN+ patients (despite both groups having been treated with ALND). However, the cNUS group had a significantly higher proportion of TNBC patients compared with the SLN+ group in addition to having higher risk features. Recognizing that TNBC patients have higher rates of distant relapse, the disparity in histologic subtypes between the two groups likely contributed to the survival differences noted. As recommendations for adjuvant systemic therapy are being tailored to tumor biology, many patients with higher risk features will be recommended for adjuvant chemotherapy regardless of nodal status.

The first interim analysis of the RxPONDER trial demonstrated that worsened tumor biology reflected in a high Oncotype Recurrence Score predicts the need for adjuvant chemotherapy in post-menopausal women with ER-positive disease, not pN1 nodal status ${ }^{23}$. The Early Breast Cancer Trialist Cooperative Group (EBCTCG) meta-analysis also highlighted the benefit of radiotherapy on locoregional recurrence and survival in patients with 1-3 positive nodes ${ }^{24}$, expanding the indication for nodal irradiation in patients with minimal nodal disease. As more patients with low nodal burden are treated with nodal irradiation and the decision for systemic chemotherapy is made based more on tumor biology than nodal status, the benefit of surgical clearance of the axilla must be re-evaluated. Additionally, for patients with ER-positive disease, options for adjuvant systemic therapies continue to expand with studies evaluating the benefit of CDK 4/6 inhibitor therapy and fulvestrant in the adjuvant setting. Clearly, there is opportunity to consider de-escalation of axillary surgery in patients who present with clinically positive nodes for whom effective adjuvant therapies exist.

Randomized trials assessing the safety of omission of ALND in patients with clinically positive nodes who are not effectively downstaged with NAC are needed. The TAXIS study, a multicenter, randomized, phase III trial is enrolling patients with clinically positive nodes to tailored axillary surgery (TAS) followed by ALND and regional nodal irradiation (RNI) or TAS followed by $\mathrm{RNI}^{25}$. The TAS procedure, as described by the investigators, involves a standard SLNB with selective removal of all palpable disease and documented removal of the biopsy-proven, clipped node. Data from our study and others confirm that a large minority of patients with clinically positive nodes have minimal nodal disease and therefore support this trial design.

We acknowledge the limitations of our study, including its retrospective design and small sample size. Many patients with clinically positive lymph nodes during this study period were treated with NAC at our institution and were excluded, thus limiting the sample size of the $\mathrm{cN}+$ patient cohort. Many of the SLN+ patients in our database who underwent ALND were considered ineligible for Z0011 based on type of 
surgery (76.6\% underwent mastectomy) rather than number of nodes involved or extranodal extension, which may result in more favorable features attributed to the SLN+ group. Furthermore, while some studies have found significant associations between presence of LVI and nodal burden ${ }^{26}$, we did not see this same effect, which may be a result of using the core needle biopsy rather than the surgical specimen to assess LVI. We purposefully chose this approach to determine whether any features identified preoperatively could be used to predict nodal disease and therefore affect nodal surgery. The absence of a direct association in our dataset could be due to the inherent risk for a false-negative result on a core biopsy and/or the small sample size. However, others have also demonstrated no correlation with LVI and nodal burden ${ }^{19,22}$.

\section{Conclusions}

In this era of effective multimodality treatment, the challenge is to identify the cohort of patients with clinically positive nodes who can be spared the morbidity of ALND. For ER-positive patients who proceed to surgery first, tumor size and lobular histology appear to be reliable predictors of higher nodal stage. Higher-risk features noted in clinically node positive patients should not preclude patients from less axillary surgery but instead guide treatment recommendations for effective adjuvant therapies. Additional studies evaluating the safety of de-escalating axillary surgery for patients with clinically positive nodes who proceed to surgery first are necessary.

\section{Declarations}

Funding Source: This study was supported by Clinical and Translational Science Institute (CTSI) Grant UL1TR000124UCLA. Dr. Linda Ye is supported in part by the HH Lee Research Program.

Conflicts of Interest / Disclosures: The authors declare that they have no conflicts of interest.

Availability of Data and Material: The datasets generated during and/or analyzed during the current study are available from the corresponding author on reasonable request

Ethics Approval: This study was reviewed and approved by the UCLA Institutional Review Board and was performed in accordance with the ethical standards as laid down in the 1964 Declaration of Helsinki and its later amendments or comparable ethical standards.

\section{Acknowledgments}

This study was supported by Clinical and Translational Science Institute (CTSI) Grant UL1TR000124UCLA. Dr. Linda Ye is supported in part by the HH Lee Research Program.

\section{References}


1. Giuliano AE, Hunt KK, Ballman KV et al (2011) Axillary dissection vs no axillary dissection in women with invasive breast cancer and sentinel node metastasis: a randomized clinical trial. JAMA Feb 9(6):569-575. doi:10.1001/jama.2011.90

2. Donker M, van Tienhoven G, Straver ME et al (2014) Radiotherapy or surgery of the axilla after a positive sentinel node in breast cancer (EORTC 10981-22023 AMAROS): a randomised, multicentre, open-label, phase 3 non-inferiority trial. Lancet Oncol Nov 15(12):1303-1310. doi:10.1016/s14702045(14)70460-7

3. Cipolla C, Valerio MR, Grassi $\mathrm{N}$ et al (Axillary nodal burden in breast cancer patients with preoperative fine needle aspiration-proven positive lymph nodes compared to those with positive sentinel nodes. In Vivo. Mar-Apr 2020);34(2):729-734. doi:10.21873/invivo.11831

4. Verheuvel NC, van den Hoven I, Ooms HW, Voogd AC, Roumen RM (2015) The role of ultrasoundguided lymph node biopsy in axillary staging of invasive breast cancer in the post-ACOSOG Z0011 trial era. Ann Surg Oncol Feb 22(2):409-415. doi:10.1245/s10434-014-4071-1

5. Caudle AS, Kuerer HM, Le-Petross HT et al (2014) Predicting the extent of nodal disease in earlystage breast cancer. Ann Surg Oncol 21:3440-7

6. Liang Y, Chen X, Tong Y et al (2019) Higher axillary lymph node metastasis burden in breast cancer patients with positive preoperative node biopsy: may not be appropriate to receive sentinel lymph node biopsy in the post-ACOSOG Z0011 trial era. World J Surg Oncol. doi.org/10.1186/s12956-019$1582-z$

7. Angarita S, Ye L, Rünger D et al (2020) Assessing the Burden of Nodal Disease for Breast Cancer Patients with Clinically Positive Nodes: Hope for More Limited Axillary Surgery. Ann of Surg Oncol /10/21 2020;doi:10.1245/s10434-020-09228-5

8. Fisher B, Jeong J-H, Anderson S et al (2002) Twenty-five-year follow-up of a randomized trial comparing radical mastectomy, total mastectomy, and total mastectomy followed by irradiation. $N$ Engl J Med. Aug 22;347(8):567-75. doi: 10.1056/NEJMoa020128

9. Johansen $\mathrm{H}$ et alExtended radical mastectomy versus simple mastectomy followed by radiotherapy in (2008) primary breast cancer. A fifty-year follow-up to the Copenhagen Breast Cancer randomised study. Acta Oncol 47:633638

10. Brinkley D, Haybittle SL (1966) Treatment of stage-II carcinoma of the female breast. Lancet. Aug 6;2(7458):291-5. doi: 10.1016/s0140-6736(66)92590-6

11. Boughey JC, Suman VJ, Mittendorf EA et al (2013) Sentinel lymph node surgery after neoadjuvant chemotherapy in patients with node-positivie breast cancer: the ACOSOG Z1071 (Alliance) clinical trial. JAMA 310:1455-1461

12. Boileau JF, Poirier B, Basik M et al (2015) Sentinel node biopsy after neoadjuvant chemotherapy in biopsy-proven node-positive breast cancer: the SN FNAC study. J Clin Oncol 33:258-264

13. Kuehn T, Banenfeind I, Fehm T et al Sentinel-lymph-node biopsy in patients with breast cancer before and after neoadjuvant chemotherapy (SENTINA a prospective multicentre cohort study (2013) Lancet Oncol 14:609-618 
14. Boughey JC, McCall LM, Ballman KV et al (2014) Tumor biology correlates with rates of breastconserving surgery and pathologic complete response after neoadjuvant chemotherapy for breast cancer: findings from the ACOSOG Z1071 (Alliance) prospective multicenter clinical trial. Ann Surg 260:608-616

15. Cortazar P, Zhang L, Untch M et al (2014) Pathologic complete response and long-term clinical benefit in breast cancer: the CTNeoBC pooled analysis. Lancet 384:164-172

16. von Minckwitz G, Untch M, Nuesch F et al (2011) Impact of treatment characteristics on response of different breast cancer phenotypes: pooled analysis of the German neo-adjuvant chemotherapy trials. Breast Cancer Res Treat 125:145-156

17. Hennessy BT, Hortobagyi GN, Rouzier R et al (2005) Outcome after pathologic complete eradication of cytologically proven breast cancer axillary node metastases following primary chemotherapy. $\mathrm{J}$ Clin Oncol 23:9304-9311

18. von Minckwitz G, Untch M, Blohmer JU et al (2012) Definition and impact of pathologic complete response on prognosis after neoadjuvant chemotherapy in various intrinsic breast cancer subtypes. J Clin Oncol 30:1796-1804

19. Harris CK, Tran HT, Lee K et al (2017) Positive ultrasound-guided lymph node needle biopsy in breast cancer may not mandate axillary lymph node dissection. Ann Surg Oncol 24:3004-3010

20. Mamtani A, Zabor EC, Stempel M, Morrow M (2019) Lobular histology does not predict the need for axillary dissection among ACOSOG Z0011-eligible breast cancers. Ann Surg Oncol 26:3269-3274

21. Lloyd P, Theophiidou E, Newcombe RG, Pugh L, Goyal A (2017) Axillary tumour burden in women with a fine-needle aspiration/core biopsy-proven positive node on ultrasonography compared to women with a positive sentinel node. BJS 104:1811-5

22. Crown A, Sevilimedu V, Morrow M (2021) Palpable Adenopathy Does Not Indicate High-Volume Axillary Nodal Disease in Hormone Receptor-Positive Breast Cancer. Ann Surg Oncol Apr 19. doi:10.1245/s10434-021-09943-7

23. Kalinsky K, Barlow WE, Meric-Bernstam F et al SWOG S1007:adjuvant trial randomized ER+ patients who had a recurrence score $<25$ and 1-3 positie nodes to endocrine therapy (ET) versus ET + chemotherapy. In: Virtual San Antonio Breast Cancer Symposium; 8-11 December 2020

24. McGale P, Taylor C, Correa $C$ et al (2014) Effect of radiotherapy after mastectomy and axillary surgery on 10-year recurrence and 20-year breast cancer mortality: meta-analysis of individual patient data for 8135 women in 22 randomised trials. Lancet Jun 21 383(9935):2127-2135. doi:10.1016/s0140-6736(14)60488-8

25. Tailored axillary surgery with or without axillary lymph node dissection followed by radiotherapy in patients with clinically node-positive breast cancer (TAXIS). ClinicalTrials.gov identifier:

NCT03513614. Updated February 15, 2021. Accessed April 7 (2021)

https://clinicaltrials.gov/show/NCT03513614

26. Pilewskie M, Mautner SK, Stempel M, Eaton A, Morrow M (2016) Does a Positive Axillary Lymph Node Needle Biopsy Result Predict the Need for an Axillary Lymph Node Dissection in Clinically 
Node-Negative Breast Cancer Patients in the ACOSOG Z0011 Era? Ann Surg Oncol Apr 23(4):1123-8. doi:10.1245/s10434-015-4944-y

Figures

A Age

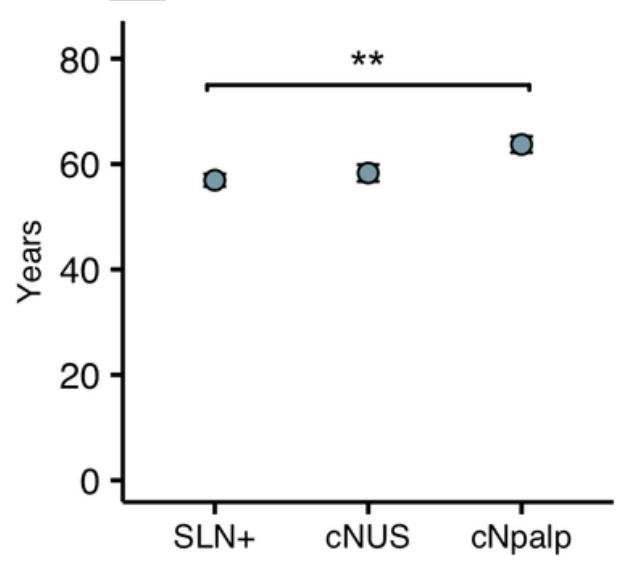

D pN1 disease

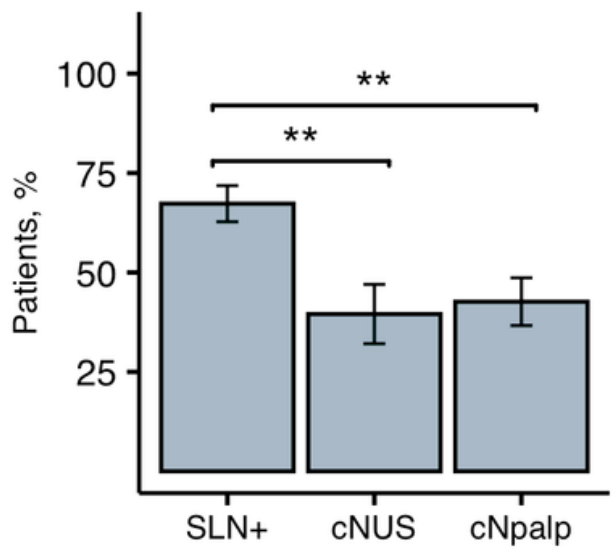

G Tumor grade

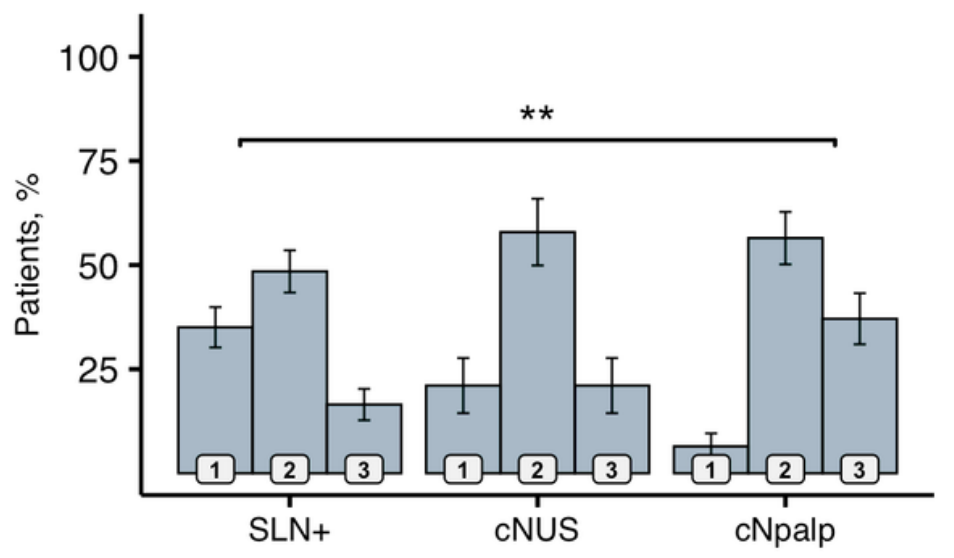

B White

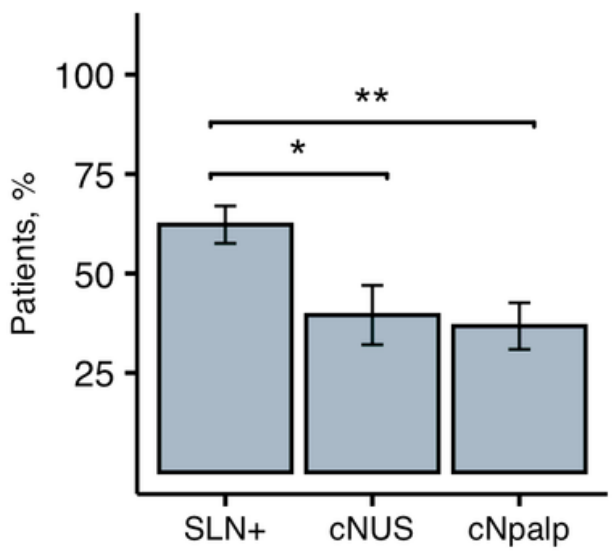

E PR positivity

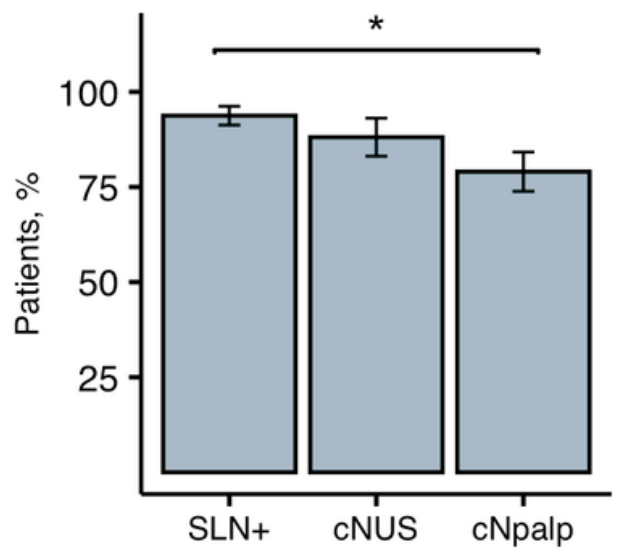

C Post-menopause

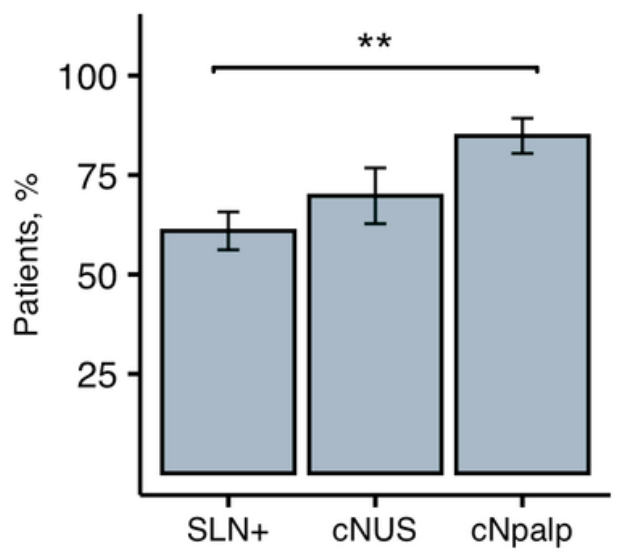

F LVI

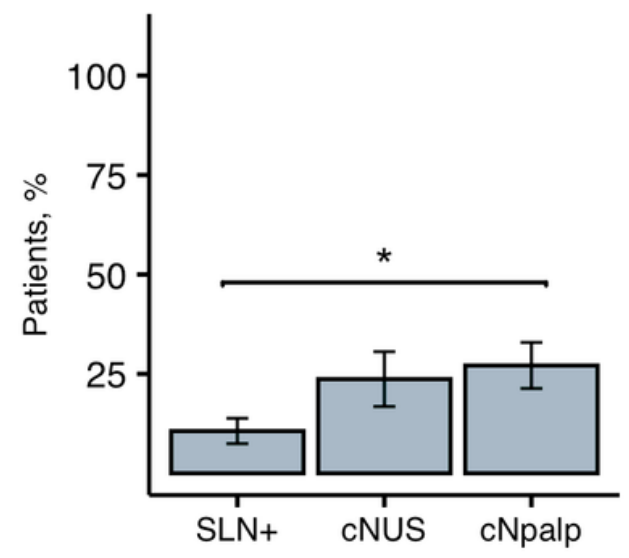

Figure 1 
Pairwise comparisons of the nodal groups SLN+, cNUS, and cNpalp for age (A), White race (B), postmenopausal status (C), pN1 disease (D), PR-positivity (E), LVI (F), tumor grade (G), and Ki67 (H). Error bars indicate \pm 1 standard error. Statistically significant group differences are shown. ${ }^{\star} p<.05 .{ }^{*} p<.01$. 